\title{
Vehicle Clustering for Improving Enhanced LTE-V2X Network Performance
}

\author{
Petri Luoto ${ }^{\star}$, Mehdi Bennis ${ }^{\star}$, Pekka Pirinen ${ }^{\star}$, Sumudu Samarakoon ${ }^{\star}$ Kari Horneman ${ }^{\dagger}$, Matti Latva-aho \\ ${ }^{\star}$ Centre for Wireless Communications \\ University of Oulu, Finland \\ ${ }^{\dagger}$ Nokia Bell Labs \\ Kaapelitie 4, \\ P.O. Box 4500, FI-90014 Oulu \{petri.luoto, \\ mehdi.bennis, pekka.pirinen, sumudu.samarakoon, \\ P.O. Box 319, FI-90620 Oulu \\ kari.horneman@nokia-bell-labs.com
}

matti.latva-aho\}@ee.oulu.fi

\begin{abstract}
Vehicle-to-Everything (V2X) communication holds the promise for improving road safety and reducing road accidents by enabling reliable and low latency services for vehicles. Vehicles are among the fastest growing type of connected devices. Therefore, there is a need for V2X communication, i.e., passing of information from Vehicle-to-Vehicle (V2V) or Vehicle-to-Infrastructure (V2I) and vice versa. In this paper, we focus on both V2I and V2V communication in a multi-lane freeway scenario, where coverage is provided by the Long Term Evolution Advanced (LTE-A) road side unit (RSU) network. Here, we propose a mechanism to offload vehicles with low signal-to-interference-plus-noise ratio (SINR) to be served by other vehicles, which have much higher quality link to the RSU. Furthermore, we analyze the improvements in the probabilities of achieving target throughputs and the performance is assessed through extensive system-level simulations. Results show that the proposed solution offloads low quality V2I links to stronger V2V links, and further increases successful transmission probability from $93 \%$ to $99.4 \%$.
\end{abstract}

Index Terms-LTE-V, V2I, V2V, vehicle, reliability, system level simulations, 5G.

\section{INTRODUCTION}

Vehicle-to-Everything (V2X) communication holds the promise for improving road safety and reducing road accidents by enabling reliable and low latency services for vehicles such as forward collision warning, road safety services and emergency stop [1]. Vehicles are among the fastest growing type of connected devices [2]. Therefore, there is a need for improving the existing Long Term Evolution Advanced (LTE-A) communication. This is particularly relevant for V2X communication, which is an important feature of LTE Release $14[3]$.

V2X communication is extremely challenging due to the fact that communication needs to be done in real-time, thus strict requirements are needed [4]. The end-to-end latency requirements of less than $5 \mathrm{~ms}$ for message sizes of about 1600 bytes need to be guaranteed for all V2X transmissions with a probability of $99.999 \%$. Traffic is either event-driven or periodically sent, with a typical time interval of $100 \mathrm{~ms}$.

This research was supported by the Finnish Funding Agency for Technology and Innovation (TEKES), Nokia, Anite, Huawei Technologies, and Infotech Oulu Graduate School.
Relative speeds up to $500 \mathrm{~km} / \mathrm{h}$ should be supported in highway scenarios.

The importance of $\mathrm{V} 2 \mathrm{X}$ communication has been recognized for years because it is considered as an important part of future Intelligent Transportation Systems (ITS). Dedicated Short-Range Communications (DSRC) has been studied for a decade and it is based on the IEEE 802.11p technology, which appeared as the most promising technique for $\mathrm{V} 2 \mathrm{X}$ communication [5], [6]. However, recent studies advocate using LTE as the preferred V2X technology [7], [8], mainly because LTE cellular network infrastructure already exists [9]. Aforementioned studies have been focusing mostly on Vehicleto-Vehicle (V2V) communication or analyzing transmission delays.

In this paper, we focus on both the Vehicle to Infrastructure (V2I) based communication and Vehicle-to-Vehicle (V2V) communication in the downlink direction. Infrastructure sends messages to vehicles for example informing the need of alternative route due to an unexpected emergency situation on the highway. In vehicular networks the reliable communication is the most important. However, especially in the cell-edge reliable communication can be challenging [10]. To ensure reliable communication, we propose that vehicles with low signal-to-interference-plus-noise ratio (SINR) are offloaded to be served by another vehicle known as a cluster leader, which has much higher quality link to the road side unit (RSU). Due to the use of high quality two-hop transmission, the need of retransmission over a single weak link can be avoided. Thus, the expected additional delay due to two-hop transmission becomes insignificant over the model of single hop transmission. In this work, the performance of a six-lane highway covered by LTE-A RSU is analyzed. We assume network without Proximity Service (ProSe) capability is investigated using an LTE-A compliant system level simulator in terms of outage probabilities reductions.

The core of the extensive LTE-A system level network simulator has been built according to the International Telecommunication Union's system level simulation guidelines [11] and calibrated and rigorously evaluated in selected macro and microcell environments [12], [13]. The simulator is extended to incorporate $\mathrm{V} 2 \mathrm{X}$ communication networks and previously 
utilized in [10].

This paper is organized as follows. The system and link models are defined in Section II. Spectral clustering for V2V communication is shown in Section III. Section IV provides the performance evaluation of the vehicular network. Finally, Section V concludes the paper.

\section{SYSTEM AND LINK MODEL}

A network with single user single-input multiple-output (SU-SIMO) with orthogonal frequency-division multiple access (OFDMA) is considered. It consists of a set of RSUs $\mathcal{B}$, each equipped with $N_{\mathrm{t}}$ transmit antennas (Tx) and a set of vehicles $\mathcal{V}$, each equipped with $N_{\mathrm{r}}$ receive antennas (Rx). The frequency domain resource consists of a set of subcarriers $\mathcal{S}$.

Let $\mathcal{T}$ be the set of transmitters, which is either $\mathcal{B}$ for V2I communication or a set of cluster leader vehicles $\mathcal{L} \subset \mathcal{V}$ for $\mathrm{V} 2 \mathrm{~V}$ communication. Furthermore, $\mathcal{V}_{t}$ is the set of vehicles served by transmitter $t \in \mathcal{T}$. In the SU-SIMO transmission scheme, the signal vector received from transmitter $t$ at $v \in \mathcal{V}_{t}$ over subcarrier $s$ is given by

$$
\mathbf{y}_{t, v}^{s}=\mathbf{h}_{t, v}^{s} \mathbf{x}_{t, v}^{s}+\sum_{i \in \mathcal{T} \backslash t} \mathbf{h}_{i, v}^{s} \mathbf{x}_{i, v}^{s}+\mathbf{n}_{t, v}^{s},
$$

where $\mathbf{x}_{t, v}^{s}$ is the transmitted signal from the transmitter $t$ to vehicle $v$ over subcarrier $s, \mathbf{h}_{t, v}^{s}$ is the channel matrix from transmitter $t$ to the $v$ th vehicle over the $s$ th subcarrier, $\mathbf{x}_{i, v}^{s}$ is the transmitted signal from the $i$ th interfering transmitter over subcarrier $s, \mathbf{h}_{i, v}^{s}$ is the channel matrix from the $i$ th interfering transmitter to the $v$ th vehicle over subcarrier $s$, and $\mathbf{n}_{t, v}^{s} \sim$ $\mathcal{C N}\left(\mathbf{0}, N_{0} \mathbf{I}_{N_{r_{v}}}\right)$ denotes the additive noise with zero mean. The maximum ratio combining (MRC) weight vector $\mathbf{w}_{t, v}^{s} \in$ $\mathbb{C}^{N_{r} \times N_{t}}$ is given by

$$
\mathbf{w}_{t, v}^{s}=\left(\mathbf{h}_{t, v}^{s}\right)^{*},
$$

where $(\cdot)^{*}$ denotes the conjugate transpose.

The entire network is modelled by using the system level simulator. Here, the link model between an RSU and a vehicle is illustrated in Fig. 1. A detailed link-to-system interface (L2S) is used in the simulations. Each vehicle is paired to an RSU based on the following path loss model

$$
\mathrm{PL}_{\mathrm{dB}}=100.7+23.5 \log _{10}(d),
$$

where $d$ is distance in kilometers. The path loss model is given in [14], where it is described as macro to relay pathloss model. When a vehicle is served by another vehicle, V2V communication path loss is defined as follows [15]

$$
\mathrm{PL}_{\mathrm{dB}}=63.3+17.7 \log _{10}(d) \text {. }
$$

In order to model fast fading and shadowing losses, a geometry-based stochastic channel model (GSCM) [11], [16] is used for all V2I links. Channel parameters are determined stochastically, based on the statistical distributions extracted from channel measurements [17]. Stochastic channel parameters are adopted from urban macro environment. For V2V communication a simple independent and identically distributed (i.i.d.) channel model is used. Carrier frequency for V2I communication is $2 \mathrm{GHz}$ and $6 \mathrm{GHz}$ for $\mathrm{V} 2 \mathrm{~V}$

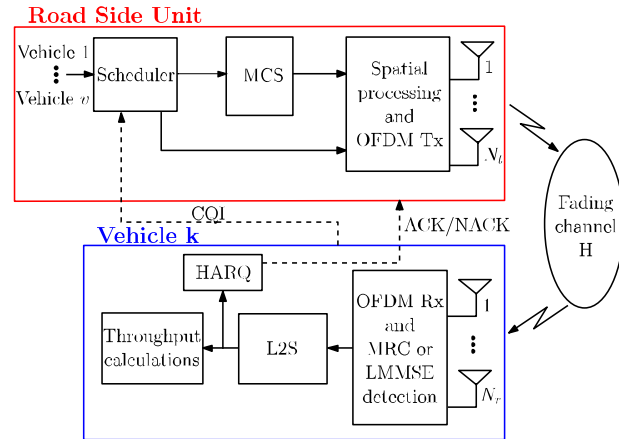

Fig. 1: Block diagram of the link model.

communication, thus there is no interference between V2I and $\mathrm{V} 2 \mathrm{~V}$ communication.

In the simulations, proportional fair (PF) scheduling is used to allocate resources for each vehicle. For V2V communication, cluster leaders use the same PF scheduler. Based on the channel-quality indicator (CQI) transmitted by the vehicle, resource allocation is performed. CQI is estimated from the received signal and for each vehicle, SINR is calculated for every physical resource block (PRB). In order to model a realistic LTE-A system, periodic and delayed CQI is assumed. For each vehicle, modulation and coding scheme (MCS) is selected based on the SINR. The cyclic prefix is assumed to be longer than the multipath delay spread, and thus, intersymbol-interference is not considered.

In the receiver side, perfect frequency and time synchronization is assumed. Link-to-system mapping is performed using mutual information effective SINR mapping (MIESM) [18]. This significantly reduces the computational overhead compared with exact modeling of the radio links, while still providing sufficiently accurate results. In the link-to-system interface, SINR is calculated and it is mapped to corresponding average mutual information.

The frame error probability (FEP) is approximated according to a predefined frame error rate (FER) curve of the used MCS. After obtaining FER values, successful and erroneous frames can be detected. Hybrid automatic repeat request (HARQ) is used for retransmissions. An acknowledgement (ACK) or a negative acknowledgement (NACK) message is sent back to the RSU to signal the success or the failure of the V2I transmission, respectively. The results are obtained by simulating a predefined number of channel samples.

\section{Performance Improvement via Spectral Clustering}

As shown in [10], the main challenge is to serve the vehicles at the cell edge, requiring a high number of PRBs due to lower SINR than vehicles at cell center with high SINR. As the network becomes dense, serving the increased number of cell edge vehicles becomes more challenging. Assuming that each vehicle can report its own geographical location to the RSU based on [19], above problem is addressed by creating clusters of low SINR vehicles, which are served by a set of vehicles known as cluster leaders. Cluster leaders have high quality 


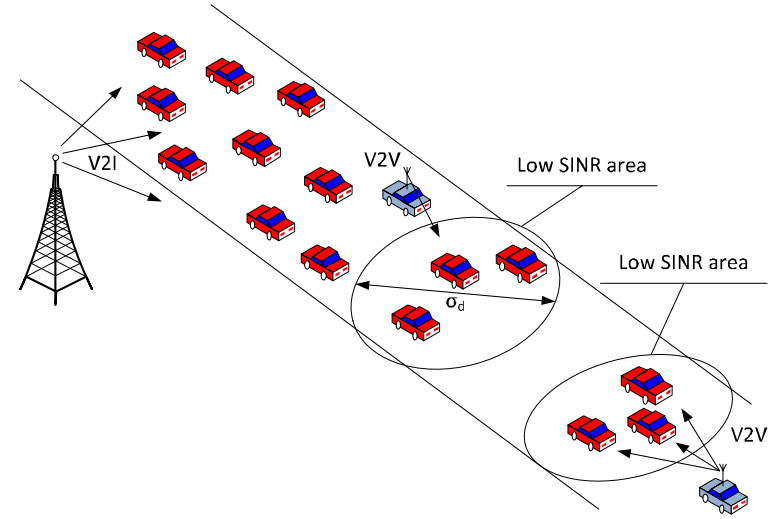

Fig. 2: V2X network with low SINR vehicle clusters and cluster leaders.

V2I connections as well as high quality V2V connections with the cluster members. Geographical information is utilized at the RSU to cluster low SINR vehicles and select cluster leaders, which are the vehicles closest to cluster centers with high quality V2I links. RSU informs above information to the vehicles. Here, clustering overhead is insignificant, due to the low cluster update frequency compared to the actual data transmissions. Fig. 2 illustrates the V2X network scenario with clustering.

Exploiting the knowledge on the location of each vehicle, RSU measures the similarity between vehicles with low SINR based on the inter-vehicular distances and groups them into multiple clusters. In this work, the similarity between two vehicles $v, v^{\prime} \in \mathcal{V}_{\text {low }}$ with their locations $y_{v}$ and $y_{v}^{\prime}$ is measured by the Gaussian similarity function as follows [20]:

$$
s_{v, v^{\prime}}=\exp \left(\frac{-\left\|y_{v}-y_{v^{\prime}}\right\|^{2}}{2 \sigma^{2}}\right)
$$

where $\sigma$ controls the impact of neighborhood size. Here, $\mathcal{V}_{\text {low }}=\left\{v \in \mathcal{V} \mid \operatorname{SINR}_{v}<\gamma_{0}\right\}$ is the set of low-SINR vehicles with SINR below a predefined threshold $\gamma_{0}$. Furthermore, the distance-based similarity matrix $\mathbf{S}$ is formed using $s_{v, v^{\prime}}$ as the $\left(v, v^{\prime}\right)$-th entry. The idea behind (5) is that the vehicles located far from each other have low similarities, and as they come closer, similarities increase in which vehicles are more likely in the same cluster. Using the similarities $\mathbf{S}$ of the low SINR vehicles, spectral clustering is performed ${ }^{1}$. Spectral clustering method exploits both the connectivity and the compactness of nodes in a graph, which is in our work is the geometry of the vehicle distribution in the network. The number of clusters $k$ is a vital input for spectral clustering. For a given neighborhood size, sparse networks consist of vehicles that are isolated from each other and thus, a large $k$ is needed. For dense networks, most of the vehicles are close to each other, and thus, a small $k$ is sufficient to provide high quality V2V links. Thus, an efficient choice of $k$ for a given neighborhood size can be presented as follows [21]:

$$
k=\arg \max _{i}\left(\zeta_{i+1}-\zeta_{i}\right), \quad i=1, \ldots,\left|\mathcal{V}_{\text {low }}\right|
$$

${ }^{1}$ For the interested readers, the details of spectral clustering can be found in $[21]$.

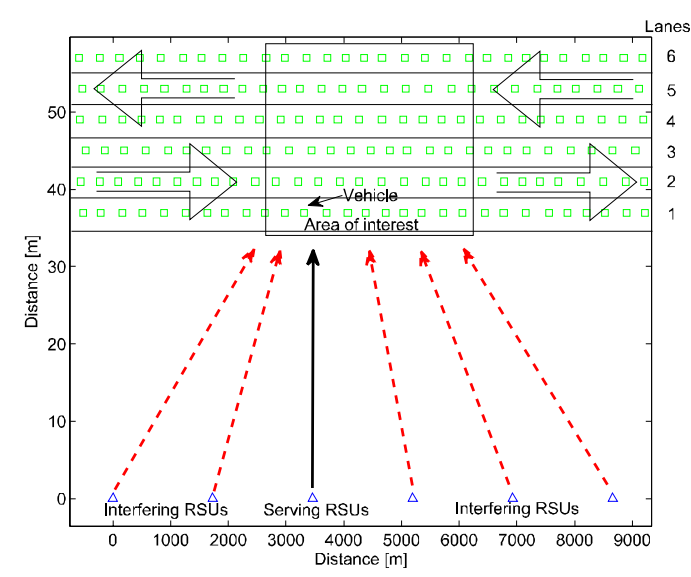

Fig. 3: Highway layout where RSUs are located next to the road.

where $\zeta_{i}$ is the $i$-th smallest eigenvalue of the network Laplacian matrix $\mathbf{L}$. Here, the network Laplacian matrix is formed at the RSU as $\mathbf{L}=\mathbf{D}-\mathbf{S}$, where $\mathbf{D}$ is the diagonal matrix with the $v$-th diagonal element as $\sum_{v^{\prime}=1}^{\left|\mathcal{V}_{\text {low }}\right|} s_{v, v^{\prime}}$. In Section IV, the impacts of the neighborhood size $\sigma$ and the SINR threshold $\gamma_{0}$ are analyzed.

\section{System LeVel Performance Results}

System level simulations are particularly useful for studying network related issues, such as resource allocation, interference management and mobility management. Moreover, 5G related system level simulations are also necessary in the future because we can not only rely on analytical analysis. Furthermore, when the simulation platform follows the standardization it can provide reliable results on the expected performance.

In this work, an LTE-A system level simulator is used to model a highway RSU network, which is used to serve vehicles moving at $140 \mathrm{~km} / \mathrm{h}$. The simulator uses a six-lane highway layout as shown in Fig. 3. Vehicles on lanes one to three are moving to the right and vehicles on lanes four to six are moving to the left. The RSU network is along the road, $35 \mathrm{~m}$ from the highway. Although all RSUs and vehicles are modeled, the performance analysis is conducted for the middle RSUs within the area of interest indicated in Fig. 3. This models the wrap-around type of interference in the system.

In order to model different vehicle densities in the network, inter vehicle distances can be varied [22]. In this work vehicles are deployed on lanes with distances from $200 \mathrm{~m}$ to $300 \mathrm{~m}$ apart from each other. Each RSU serves multiple vehicles and one vehicle is connected to a single RSU. Generally, V2X traffic is periodically sent. However, for this work we have mapped 1600 byte package to be sent with a time interval of $100 \mathrm{~ms}$ to be equivalent to a constant transmission with a target rate of $128 \mathrm{~kb} / \mathrm{s}$ per vehicle. Table I summarizes the main simulation parameters.

First, we analyze the impact of the neighborhood size $\sigma$ in Fig. 4. Here, the minimum and maximum distances between vehicles are $200 \mathrm{~m}$ to $300 \mathrm{~m}$, which correspond to around 40 
TABLE I: Simulator parameters and assumptions.

\begin{tabular}{|l|l|}
\hline Parameter & Assumption \\
\hline Duplex mode & FDD \\
\hline System bandwidth & $10 \mathrm{MHz}$ \\
\hline Number of PRBs & 50 \\
\hline $\begin{array}{l}\text { Antenna configuration } \\
\text { Vehicle speed }\end{array}$ & $\begin{array}{l}1 \mathrm{Tx} \times 2 \mathrm{Rx}, \\
140 \mathrm{~km} / \mathrm{h}\end{array}$ \\
\hline Inter vehicle distances & $\begin{array}{l}\mathrm{min} 200 \text { meters and max 300 me- } \\
\text { ters }\end{array}$ \\
\hline Inter RSU distance & $1732 \mathrm{~m}$ \\
\hline HARQ & Chase combining \\
\hline Transmission power & $46 \mathrm{dBm}$ \\
\hline Feedback CQI period & $6 \mathrm{~ms}$ \\
\hline Feedback CQI delay & $2 \mathrm{~ms}$ \\
\hline Channel estimation & Ideal \\
\hline Network synchronization & Synchronized \\
\hline Receiver type & MRC \\
\hline L2S interface metric & MIESM \\
\hline Traffic model & $\begin{array}{l}\text { Continuous constant rate transmis- } \\
\text { sion }\end{array}$ \\
\hline Scheduler & Proportional fair \\
\hline Target rate & $128 \mathrm{~kb} / \mathrm{s}$ \\
\hline
\end{tabular}

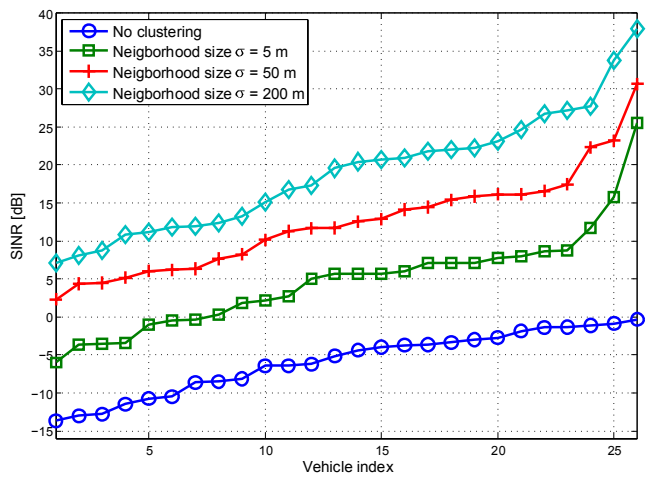

Fig. 4: Impact of the neighborhood size for $\gamma_{0}=+3 \mathrm{~dB}$.

vehicles being connected to each RSU. When the neighborhood size is 5 meters, there are only a few vehicles per cluster due to the low similarities among vehicles as per 5 . Here, the number of clusters is $k=8$ and inter cluster interference lowers the performance. For each cluster the cluster leader is the one having SINR over the threshold and being the closest to cluster center. When $\sigma_{d}=50 \mathrm{~m}$, there are $k=5$ clusters in the network and the performance is improved. For neighborhood size of 200 meters, there are $k=3$ clusters in the network, which gives the best performance due to the low inter-cluster interference. Further increasing $\sigma_{d}$ does not impact the performance. The reason is that the clusters remain unchanged, since we assume that the there is no viable V2V communication between vehicles with inter-vehicular distance above 200 m, i.e., $s_{v, v^{\prime}}=0$ if $\left\|y_{v}-y_{v^{\prime}}\right\|>200$. It should be noted that neighborhood size should be always mapped to the vehicle densities, e.g., during the rush hour $\sigma_{d}$ should be different than during the night. When network is dense and data requirement is high, larger $\sigma_{d}$ could lead to overloaded clusters, whereby cluster leaders may not be able to serve their cluster members.

Figures 5 and 6 illustrate the SINR distribution (heat map) of the network without and with clusters, respectively, over

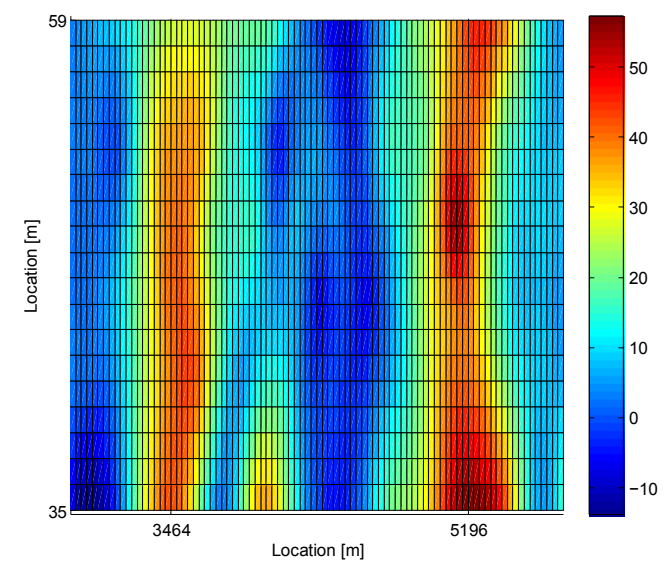

Fig. 5: SINR heat map without clustering $\left(\gamma_{0}=+3 \mathrm{~dB}, \sigma_{d}=\right.$ $200 \mathrm{~m})$.

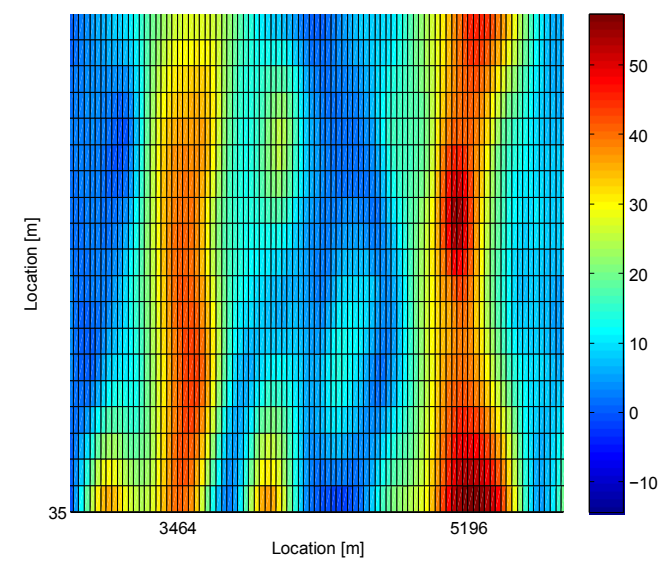

Fig. 6: SINR heat map with clustering $\left(\gamma_{0}=+3 \mathrm{~dB}, \sigma_{d}=\right.$ $200 \mathrm{~m})$.

the area of interest indicated in Fig. 3. The network without clusters in Fig. 5 exhibits two high SINR areas close to the RSU locations (at $3464 \mathrm{~m}$ and $5196 \mathrm{~m}$ ) and low SINR area in the cell edge. The proposed solution clusters these low-SINR vehicles and serves them with the aid of cluster heads resulting in improved SINR as illustrated in Fig. 6. Furthermore, it can be noted that the low SINR area disappears and SINR is increased from $-10 \mathrm{~dB}$ to $+10 \mathrm{~dB}$. This heat map clearly shows that the proposed clustering based $\mathrm{V} 2 \mathrm{~V}$ offloading greatly improves the SINR at cell edges. The dark blue (low SINR) areas disappear and the light blue/yellow (higher SINR) areas appear when spectral clustering is used.

In Fig. 7, cumulative distribution function (CDF) of the throughput of the V2X network is analyzed. Fig. 7 shows that when clustering is performed, performance is significantly increased and probability to achieve the target throughput increases from $93 \%$ to $99.4 \%$. Moreover, noticeable throughput gains can be seen as clustering update interval reduces from $1000 \mathrm{~ms}$ to $100 \mathrm{~ms}$.

Finally in Table II, the impact of the SINR threshold $\gamma_{0}$ is 


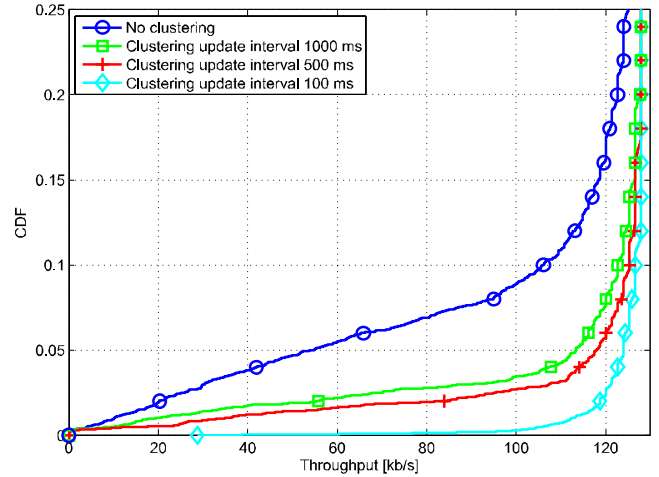

Fig. 7: Impact of the clustering update interval $\left(\gamma_{0}=+3 \mathrm{~dB}\right.$, $\left.\sigma_{d}=200 \mathrm{~m}\right)$.

analyzed. This threshold defines the set of low SINR vehicles, i.e., whether to operate as V2I link or to switch into V2V link. It can be noted that clustering vehicles even with a low SINR threshold $\gamma_{0}=-3 \mathrm{~dB}$ results in a performance gain over the no clustering scenario. Further increasing $\gamma_{0}$ from $-3 \mathrm{~dB}$ to 0 $\mathrm{dB}$ does not provide significant gain. Even though each vehicle has a good connection not all vehicles meet their target rate. The reason is that there is a possibility to have frame errors, which leads to retransmissions and thus, achieving rates below the target rates.

TABLE II: Probability to achieve a target throughput with different SINR thresholds for $\sigma_{d}=200 \mathrm{~m}$.

\begin{tabular}{|c|c|c|}
\hline No clustering & $\gamma_{0}=-3 \mathrm{~dB}$ & $\gamma_{0}=0 \mathrm{~dB}$ \\
$93.0 \%$ & $99.0 \%$ & $99.4 \%$ \\
\hline
\end{tabular}

\section{CONCLUSION}

We have evaluated the performance of Vehicle-toInfrastructure (V2I) based communication with additional Vehicle-to-Vehicle (V2V) communication in a freeway scenario. The framework has been established under the LTEA compliant system simulation platform. We analyzed the improvement of cell edge vehicle performances by offloading them to vehicles with high quality V2I links. Provided numerical results show that we can significantly improve the overall performance when spectral clustering is used. In our future work we will consider more sophisticated heterogeneous traffic models. Moreover, we will study Vehicle-to-Everything (V2X) related communication problems, exploiting Proximity Service (ProSe) capabilities.

\section{REFERENCES}

[1] "3GPP TR 36.885 V0.4.0 Study on LTE-based V2X Services." [Online]. Available: http://www.3gpp.org/dynareport/36885.htm
[2] A. Khelil and D. Soldani, "On the suitability of Device-to-Device communications for road traffic safety," in 2014 IEEE World Forum on Internet of Things (WF-IoT), March 2014, pp. 224-229.

[3] "3GPP News, LTE support for the connected car." [Online] Available: http://http://www.3gpp.org/news-events/3gpp-news/1675-lte_ automotive

[4] "METIS deliverable D1.1. Scenarios, requirements and KPIs for 5G mobile and wireless system." [Online]. Available: https://www. metis2020.com/documents/deliverables/

[5] L. Le, A. Festag, R. Baldessari, and W. Zhang, "Vehicular wireless shortrange communication for improving intersection safety," IEEE Commun. Mag., vol. 47, no. 11, pp. 104-110, 2009.

[6] R. Protzmann, B. Schunemann, and I. Radusch, "The influences of communication models on the simulated effectiveness of V2X applications," IEEE Commun. Mag., vol. 49, no. 11, pp. 149-155, 2011.

[7] M. Botsov, M. Klugel, W. Kellerer, and P. Fertl, "Location-based resource allocation for mobile D2D communications in multicell deployments," in 2015 IEEE International Conference on Communication Workshop (ICCW), 2015, pp. 2444-2450.

[8] C. Lottermann, M. Botsov, P. Fertl, and R. Mullner, "Performance evaluation of automotive off-board applications in LTE deployments," in Vehicular Networking Conference (VNC), 2012 IEEE, Nov. 2012, pp. 211-218.

[9] J. Yoshida, "Prelude to 5G: Qualcomm, Huawei Muscle into V2X," Oct. 2015. [Online]. Available: http://www.eetimes.com/document.asp? doc_id=1328030\&page_number $=2$

[10] P. Luoto, M. Bennis, P. Pirinen, S. Samarakoon, K. Horneman, and M. Latva-aho, "System level performance evaluation of LTE-V2X network," in European Wireless Conference 2016, May 2016.

[11] "WINNER II channel models, D1.1.2 V1.0." [Online]. Available: http://www.cept.org/files/1050/documents/winner2\%20-\%20final\% 20report.pdf

[12] H. Pennanen, T. Haataja, J. Leinonen, A. Tölli, and M. Latva-aho, "System level evaluation of TDD based LTE-Advanced MIMO-OFDMA systems," in IEEE GLOBECOM Workshops (GC Wkshps), Dec. 2010, pp. 809-813.

[13] T. Haataja, H. Pennanen, J. Leinonen, A. Tölli, and M. Latva-aho, "Space-frequency scheduling in TDD based LTE-Advanced MIMOOFDMA systems," in IEEE 73rd Vehicular Technology Conference (VTC Spring), May 2011, pp. 1-5.

[14] "3GPP TR 36.814 V1.7.0. Further Advancements for E-UTRA Physical Layer Aspects (Release 9).” [Online]. Available: http: //www.3gpp.org/dynareport/36814.htm

[15] J. Karedal, N. Czink, A. Paier, F. Tufvesson, and A. F. Molisch, "Path loss modeling for vehicle-to-vehicle communications," IEEE Trans. Veh. Technol., vol. 60, no. 1, pp. 323-328, Jan. 2011.

[16] "3rd Generation Partnership Project, Technical Specification Group Radio Access Network, Spatial channel model for Multiple Input Multiple Output (MIMO) simulations, 3GPP Technical report 25.996 v6.1.0.”

[17] "Guidelines for evaluation of radio interface technologies for IMTAdvanced, Report ITU-R M.2135-1."

[18] X. He, K. Niu, Z. He, and J. Lin, "Link layer abstraction in MIMO OFDM system," in International Workshop on Cross Layer Design (IWCLD), Sep. 2007, pp. 41-44.

[19] "3GPP TSG RAN WG1 Meeting \#84 Centralized Resource Allocation for V2X over PC5." [Online]. Available: http: //www.3gpp.org/DynaReport/TDocExMtg--R1-84--31660.htm

[20] A. L. Yuille and N. M. Grzywacz, "The motion coherence theory," in Second International Conference on Computer Vision, , Dec 1988, pp. 344-353.

[21] U. von Luxburg, "A tutorial on spectral clustering," CoRR, vol. abs/0711.0189, 2007. [Online]. Available: http://arxiv.org/abs/0711.0189

[22] "3GPP TSG RAN WG1 Meeting \#82 R1-153800. Overview of V2X evaluation methodology." [Online]. Available: http://www.3gpp.org/ftp/ tsg_ran/WG1_RL1/TSGR1_82/Docs/ 